\title{
Electromagnetic induction heating as a driver of volcanic activity on massive rocky planets
}

\author{
Kristina Kislyakova ${ }^{1}$ and Lena Noack ${ }^{2}$ \\ 1 University of Vienna, Department of Astrophysics, Türkenschansstrasse 17, 1180 Vienna, Austria \\ e-mail: kristina.kislyakova@univie.ac.at \\ ${ }^{2}$ Freie Universität Berlin, Malteserstrasse 74-100, 12249 Berlin, Germany
}

Received 11 March 2020 / Accepted 15 April 2020

\begin{abstract}
Aims. We investigate possible driving mechanisms of volcanic activity on rocky super-Earths with masses exceeding 3-4 $M_{\oplus}$. Due to high gravity and pressures in the mantles of these planets, melting in deep mantle layers can be suppressed, even if the energy release due to tidal heating and radioactive decay is substantial. Here we investigate whether a newly identified heating mechanism, namely induction heating by the star's magnetic field, can drive volcanic activity on these planets due to its unique heating pattern in the very upper part of the mantle. In this region the pressure is not yet high enough to preclude the melt formation.

Methods. Using the super-Earth HD $3167 \mathrm{~b}$ as an example, we calculate induction heating in the planet's interiors assuming an electrical conductivity profile typical of a hot rocky planet and a moderate stellar magnetic field typical of an old inactive star. Then we use a mantle convection code (CHIC) to simulate the evolution of volcanic outgassing with time.

Results. We show that although in most cases volcanic outgassing on HD 3167b is not very significant in the absence of induction heating, including this heating mechanism changes the picture and leads to a substantial increase in the outgassing from the planet's mantle. This result shows that induction heating combined with a high surface temperature is capable of driving volcanism on massive super-Earths, which has important observational implications.
\end{abstract}

Key words. planets and satellites: atmospheres - planet-star interactions - planets and satellites: individual: HD 3167b methods: numerical

\section{Introduction}

Volcanic outgassing is one of the major processes filling a planet's atmosphere with gases. An atmosphere can also be produced during the planet's accretion phase by rapid outgassing from the interior during the solidification of a magma ocean caused by giant impacts. If the surface temperature is high, a magma ocean can be produced via strong irradiation from the host star, as has been proposed for the planet $55 \mathrm{Cnc}$ e (Demory et al. 2016). If stellar short wavelength radiation and wind lead to rapid loss of the atmosphere to space, the atmosphere has to be replenished by volcanic outgassing (e.g., Godolt et al. 2019). This is especially relevant for close-in planets with an efficient atmospheric escape. In this paper, we focus on atmosphere formation due to volcanic activity.

Sufficient energy release inside a planet's mantle can melt it and drive very strong volcanism. As an example, Io's tidal surface heat flux of $2 \mathrm{~W} \mathrm{~m}^{-2}$ makes it the most volcanically active body in the solar system despite its relatively small size (McEwen et al. 1998). The presence and composition of a planet's atmosphere play a very important role for the atmospheric characterization. With the upcoming observational facilities such as the Atmospheric Remote-sensing Infrared Exoplanet Large-survey (ARIEL) and the James Webb Space Telescope (JWST), understanding the possible formation mechanisms of atmospheres is of pivotal importance.

Recently, Noack et al. (2017) and Dorn et al. (2018) have shown that at low masses (below 3-4 $M_{\oplus}$ ) outgassing positively correlates with the planet's mass since it is controlled by the mantle's volume. At higher masses, outgassing decreases with the planet's mass, which is due to the increasing pressure gradient that limits melting to shallower depths. Observations of small rocky exoplanets with masses lower than 3-4 $M_{\oplus}$ are extremely challenging. Although ARIEL will be able to observe some small planets, most of its rocky targets will be more massive than the Earth (e.g., Edwards et al. 2019). Therefore, many planets in the ARIEL and JWST target lists can be potentially affected by insufficient outgassing, and thus a lack of observable atmosphere. In this paper we investigate whether a newly identified heating mechanism, namely heating by electromagnetic induction recently suggested by Kislyakova et al. (2017), can change this pattern.

Induction effects arise when a planet is embedded in a varying stellar magnetic field and influence the entire planet, from the interior to the atmosphere, and can have observable effects. Induction heats the planet by alternating currents generated in the planetary mantle and drives strong volcanic activity. For planets orbiting $\mathrm{M}$ dwarfs with magnetic fields of a few hundred gauss or more or for planets orbiting very close to stars with solar-like magnetic fields, induction heating of the interiors can be as powerful an energy source as tidal heating (Kislyakova et al. 2018; Günther \& Kislyakova 2020). In this paper, we focus on rocky super-Earths orbiting close to their host stars with moderate magnetic fields. This paper is organized as follows: Sect. 2 briefly describes our methods, Sect. 3 presents our results, and Sect. 4 summarizes our conclusions. 
Table 1. Parameters of the star HD 3167 and planet HD 3167b adopted from Gandolfi et al. (2017).

\begin{tabular}{|c|c|}
\hline Parameter & Value \\
\hline Stellar mass $\left(M_{\odot}\right)$ & $0.877 \pm 0.024$ \\
\hline Stellar radius $\left(R_{\odot}\right)$ & $0.835 \pm 0.026$ \\
\hline Stellar age (Gyr) & $5.0 \pm 4.0$ \\
\hline Stellar equilibrium temperature $(\mathrm{K})$ & $5286 \pm 40$ \\
\hline Stellar rotational period (days) & $23.52 \pm 2.87$ \\
\hline Stellar magnetic field ${ }^{(1)}(\mathrm{G})$ & $1-10$ \\
\hline Planetary mass $\left(M_{\oplus}\right)$ & $5.69 \pm 0.44$ \\
\hline Planetary radius $\left(R_{\oplus}\right)$ & $1.574 \pm 0.054$ \\
\hline Planetary equilibrium temperature $(\mathrm{K})$ & $1759 \pm 20$ \\
\hline Semi-major axis $(\mathrm{au})$ & $0.01752 \pm 0.00063$ \\
\hline Orbital inclination ${ }^{(2)}$ (degree) & 85 \\
\hline
\end{tabular}

Notes. ${ }^{(1)}$ Following the magnetism trends presented by Vidotto et al (2014). ${ }^{(2)}$ Between the stellar rotational axis and the planet's orbital plane.

\section{Methods}

For induction heating to be substantial, two factors are necessary: a strong magnetic field at the planet's orbit and a periodic variation of the amplitude of this field. The period of the field variation is determined by the star's rotation, by the planet's orbital period, or by a combination of the two. The star's rotation leads to induction effects in planets if the star's magnetic dipole axis is inclined with respect to its rotation axis. Observations and magnetic maps show that any angle between the two axes is possible (Lang et al. 2012; Morin et al. 2010; Fares et al. 2013, 2017). In this study, we investigate the induction effects in HD $3167 \mathrm{~b}$, which is a transiting super-Earth with a mass of $5.7 M_{\oplus}$ and a radius of $1.57 R_{\oplus}$. Interesting features of this planet are its polar orbit and its close distance to the host star, which are prerequisites for efficient induction heating and resulting volcanic activity. Parameters of the system are summarized in Table 1. We assume that the star's magnetic dipole axis coincides with the star's rotation axis. The inclination of the orbit of HD $3167 \mathrm{~b}$ with respect to the star's rotation axis is unknown; however, Dalal et al. (2019) found that the second planet in the system, HD 3167c, is on a nearly polar orbit. Since most planetary systems are coplanar and both planets are transiting, HD 3167b should also have a polar orbit. We assume an orbital inclination of $85^{\circ}$. Such high orbital inclination can cause a strong induction heating due to a variation of the star's magnetic field at the planet's location due to the planet's motion around the star (Kislyakova et al. 2018). To calculate the magnetic field at a given orbital distance, we use a potential field source-surface model, which is commonly used to model stellar magnetic fields (Johnstone 2012), as described in Kislyakova et al. (2017, 2018).

We use the code developed to calculate induction heating in planetary interiors, which has been applied to exoplanets with Earth-like conductivity profiles (Kislyakova et al. 2017, 2018; Günther \& Kislyakova 2020). The code is based on the method presented in Parkinson (1983) for a sphere with a non-uniform electrical conductivity. The code divides the planetary mantle into layers and solves the induction equation in every layer to find the magnetic field and current. The energy release within each layer can be determined from the current and conductivity. For a simple stellar dipole field, the field around a planet can be considered uniform due to stretching of the magnetic field lines by the stellar wind. Then the current flowing inside the planet has

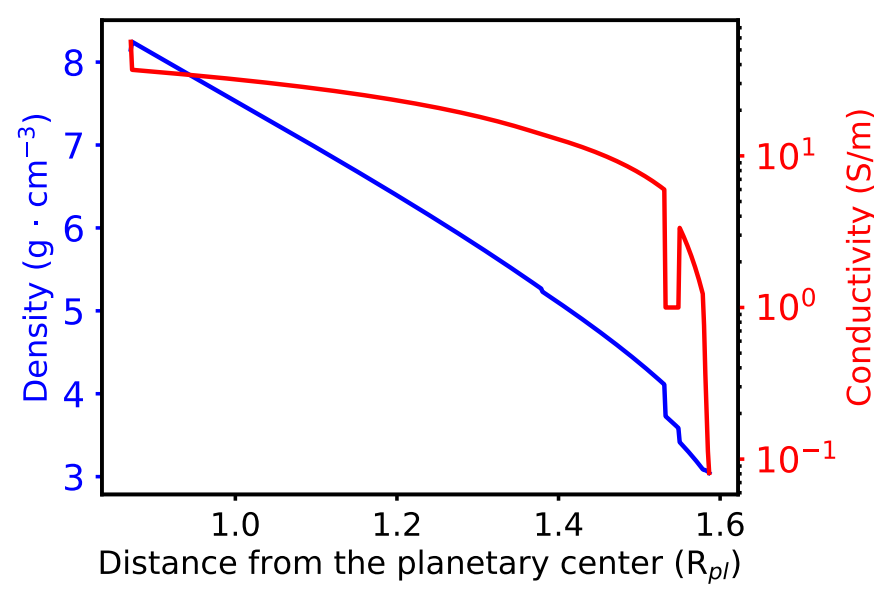

Fig. 1. Density and conductivity profiles of the super-Earth HD 3167b, assuming a composition of $40 \%$ iron and $60 \%$ silicates and a surface temperature of $1759 \mathrm{~K}$ calculated with the code CHIC (Noack et al. 2016). For comparison, the Earth has an iron content of about $32 \%$. The profile is adopted from Günther \& Kislyakova (2020).

a simple form of a circular current that is the strongest near the planet's equator, where the equator is defined by the direction of the stellar magnetic field. The magnitude of the current and the heating decreases both with depth and latitude, as is typical of the skin effect.

The conductivity profile of a planet's mantle is an important factor that influences the energy release by induction heating. Electrical conductivity and the frequency of variation of the magnetic field determine the skin depth, $\delta$, which is the penetration depth of the electromagnetic field into the medium, defined as the level where its amplitude decreases by a factor of $e$. In the approximation of a highly conductive medium $(4 \pi \sigma \gg \epsilon \omega)$, which is applicable for the parameter range of interest, the skin depth is given by $\delta=c / \sqrt{2 \pi \sigma \mu \omega}$, where $c$ is the speed of light, $\sigma$ is the electrical conductivity of the medium, $\mu$ is the magnetic permeability, $\epsilon$ is the permittivity, and $\omega$ is the frequency of the field change. We assume $\mu=\epsilon=1$ because the Curie temperature at which the materials lose their magnetic properties (about $600^{\circ}$ for olivine) is reached at a depth of only $20-90 \mathrm{~km}$ in our model, and this depth depends on the assumed planet and mantle parameters (see discussion in Kislyakova et al. 2017). The skin depth drastically decreases in the deeper layers, meaning that the varying magnetic field cannot penetrate the entire volume of the mantle. The conductivity is given by

$\sigma=\sigma_{0} \exp \left(-\frac{\Delta H}{k_{\mathrm{B}} T}\right)$

where $k_{\mathrm{B}}$ is the Boltzmann constant and $T$ the local temperature. The values of $\sigma_{0}$ and $\Delta H$ for different mineral phases are taken from experimental studies (Xu et al. 2000; Yoshino et al. 2008; Yoshino \& Katsura 2013). Figure 1 shows density and conductivity profiles calculated for HD 3167b with the interior structure module in the code Coupling Habitability, Interior, and Crust (CHIC; Noack et al. 2016). We assumed an Earth-like mantle mineralogy composed of $\mathrm{Mg}, \mathrm{Fe}, \mathrm{Si}$, and $\mathrm{O}$, which make up most of the Earth's mantle. Phase transitions (olivine $\rightarrow$ wadsleyite, wadsleyite $\rightarrow$ ringwoodite, ringwoodite $\rightarrow$ perovskite mixed with magnesiowustite, and perovskite-post-perovskite) are clearly visible in the interior profile shown in Fig. 1.

We calculated the amount of outgassing from the mantle of HD 3167b using the 2D mantle convection module in CHIC 
(Noack et al. 2016). The code models the mantle convection and related magmatic events in a regional 2D spherical annulus geometry for a compressible mantle (Noack et al. 2017). It also traces the amount of melt and its evolution in time.

Here we present one example case to demonstrate the effect of the induction heating. The core was initially super-heated and cooled over time. We added Earth-like decaying radiogenic heat sources that heat the mantle. We assumed that the planet is in the stagnant lid regime. Plate tectonics would allow for strong outgassing; however, a stagnant lid is more likely for HD $3167 \mathrm{~b}$ due to its high surface temperature (Lenardic et al. 2008). We set the surface temperature in the convection simulations to $1000 \mathrm{~K}$, which is lower than the equilibrium temperature of HD 3167b of $1759 \mathrm{~K}$. A higher surface temperature cannot be modeled due to numerical constraints. Since HD $3167 \mathrm{~b}$ is likely tidally locked, the temperature of $1759 \mathrm{~K}$ may be reached only in some regions of one hemisphere. Therefore, a surface temperature of $1000 \mathrm{~K}$ is a reasonable approximation for the global average temperature. Simulations start directly after the solidification of the magma ocean.

Furthermore, we assume that initially $200 \mathrm{wt}$-ppm of water are stored in the mantle, and consider a moderately oxidized mantle $(\mathrm{IW}+2)$ with initially $100 \mathrm{wt}-\mathrm{ppm} \mathrm{CO}_{2}$ being stored in the mantle in the form of graphite. The main outgassing products in our simulations are $\mathrm{H}_{2}, \mathrm{H}_{2} \mathrm{O}, \mathrm{CO}$, and $\mathrm{CO}_{2}$ based on the local oxygen fugacity and volatile abundances in the melt (Ortenzi et al. 2019).

\section{Results}

Figure 2 shows the calculated induction heating inside the planetary mantle assuming different dipole strengths of the global magnetic field of the star HD 3167 of 1, 5, and 10 gauss. Young fast rotating Sun-like stars can generate strong global magnetic fields reaching around $100 \mathrm{G}$. As stars age their rotation decelerates and their magnetic fields become weaker (Güdel 2007; Vidotto et al. 2014). Older Sun-like stars also exhibit cyclic behavior when the strengths of their magnetic fields and their dipole components vary periodically, with periods of years (Fares et al. 2013, 2017; Boro Saikia et al. 2018). However, if a planet orbits very close to its host star, even an older star with a global dipole component of 3-10 G can generate a magnetic field of $\sim 0.1-0.5 \mathrm{G}$ at the planet's orbit. A global dipole field of 10 gauss and less is typical for old and inactive stars such as HD 3167, which has a rotation period of 26 days (Vidotto et al. 2014). For comparison, the global solar dipole field varies in the range of $1-7 \mathrm{G}$. The magnetic field at the planet's orbit was calculated as in Kislyakova et al. (2017) and was equal to 0.05, 0.26 , and $0.52 \mathrm{G}$ for the star's global dipole field of 1,5 , and 10 gauss, respectively. The total energy release due to induction heating in these three cases was equal to $1.8 \times 10^{19}, 4.5 \times 10^{20}$, and $1.8 \times 10^{21} \mathrm{erg} \mathrm{s}^{-1}$, respectively. As one can see, the energy release scales with the magnetic field to the power of two.

Figure 3 shows the evolution of the total amount of volcanic outgassing for a global dipole field of HD 3167 of 0 (no induction heating), 1, 5, and 10 gauss, assuming Earth-like mantle viscosity (left panel) and viscosity enhanced by a factor of 100 (following Tackley et al. 2013; right panel). Even a moderate global stellar magnetic field of 5 gauss leads to a much earlier onset of the volcanic activity on the planet and significantly increases its magnitude in comparison to the case without induction heating ( 0 gauss). In the 0 gauss case, heat is still produced in the mantle due to radioactive decay. In the case with increased viscosity without induction heating, it takes more than

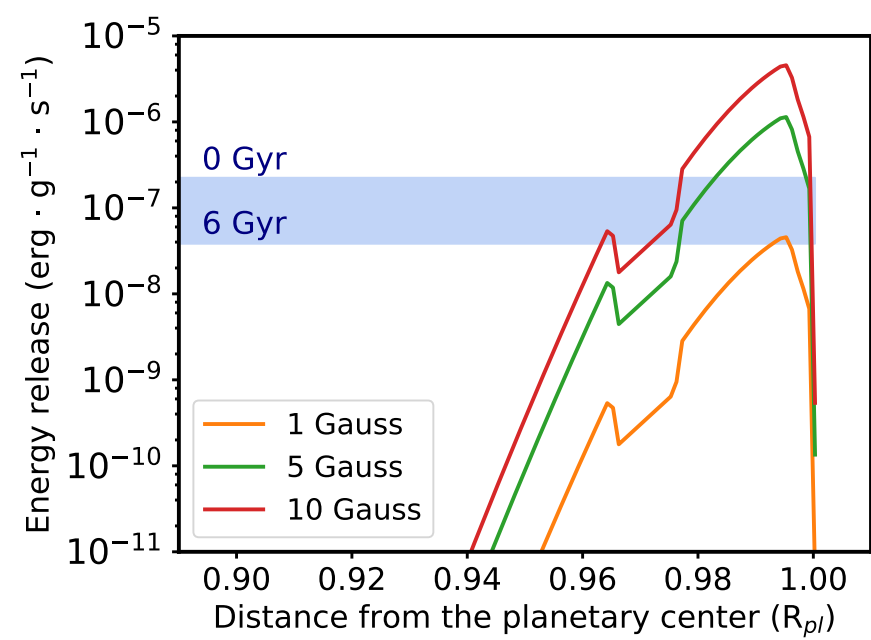

Fig. 2. Energy release inside the planet HD $3167 \mathrm{~b}$ assuming a conductivity profile with an iron mass fraction of $40 \%$ and various strengths of the stellar magnetic field. The orbital inclination is $85^{\circ}$. The energy is released in the upper $10 \%$ of the planet's mantle. The shaded region shows the radiogenic heat sources at the formation time of the system (upper limit) and at the age of $6 \mathrm{Gyr}$ (lower limit).

five gigayears for the outgassed atmosphere to accumulate a pressure of several bars. In that case, due to a thick lithosphere that forms in the absence of plate tectonics, only a small amount of melt can form and rise to the surface due to the high pressure at the bottom of the lithosphere (Dorn et al. 2018). For an Earthlike viscosity, outgassing is possible due to high surface temperature and efficient mantle convection. However, induction heating increases the outgassing by several dozen bar, if the global stellar magnetic field exceeds 5 gauss.

All cases that include induction heating show an earlier onset of volcanic activity and predict the formation of an atmosphere with a pressure of several tens of bars. Even a weak stellar magnetic field of 1 gauss leads to a minor increase in outgassing at later evolutionary stages, when the radiogentic heat sources decline. Considering the close proximity of HD $3167 \mathrm{~b}$ to its host star, the atmosphere of this planet is likely thinner than the estimates shown in Fig. 3 because these results do not take into account escape to space or any other atmospheric sinks. Global magnetic fields of 5 to 10 gauss are quite typical for stars with activity levels and ages similar to HD 3167 (Vidotto et al. 2014). In summary, our results predict active volcanism and the presence of an atmosphere or at least an extended exosphere on HD $3167 \mathrm{~b}$ in contrast to earlier studies that neglected induction heating (Noack et al. 2017; Dorn et al. 2018).

Exoplanets on inclined orbits are subject to tidal heating. However, the local energy release by tidal dissipation depends on the rheology and shear modulus the mantle (Roberts \& Nimmo 2008). More energy is released in the lower mantle where the temperatures are higher than in the upper mantle. Therefore, tidal heating is unlikely to melt the mantle of a planet as massive as HD 3167b. Unlike tidal heating, induction heating preferentially heats the very upper part of the mantle. Our results confirm that in this case some melt is produced in the upper mantle, resulting in volcanic activity. From our simulations we see that induction heating is indeed a possible driver of volcanism on super-Earths, and that it is very efficient for close-in planets orbiting Sun-like stars. Another important contributing factor is likely the high surface temperature, which makes the lithosphere thinner, which is why even in the case without induction heating 

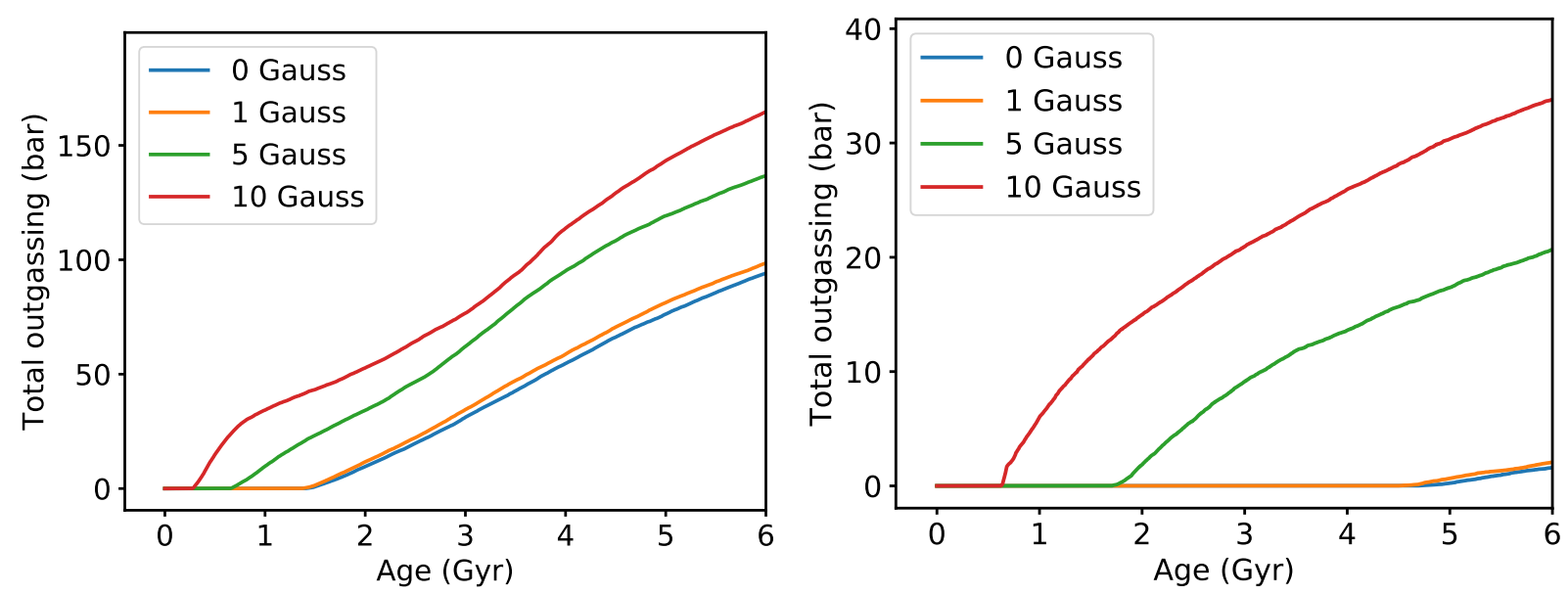

Fig. 3. Total outgassing of $\mathrm{CO}_{2}, \mathrm{CO}, \mathrm{H}_{2} \mathrm{O}$, and $\mathrm{H}_{2}$ from $\mathrm{HD} 3167 \mathrm{~b}$ assuming the heating shown in Fig. 2 for the magnetic field of the host star of $0,1,5$, and $10 \mathrm{G}$. Induction heating leads to a much earlier onset of volcanism on the planet and increases the outgassing by several tens of bar. Left panel: low (Earth-like) viscosity case. Right panel: mantle viscosity is increased by a factor of 100 .

some outgassing can occur in the stagnant lid regime, in contrast to the predictions by Noack et al. (2017) and Dorn et al. (2018).

\section{Conclusions}

Our simulations confirm the suggestion by Günther \& Kislyakova (2020) that induction heating can enhance volcanic activity in massive super-Earths. These findings have very important implications for observational missions such as ARIEL, JWST, and the ground-based facilities if future spectroscopical observations of these massive exoplanets reveal the presence of atmospheres or exospheres with compositions typical of volcanic outgassing. Previously, some signs of volcanic activity were detected in 55 Cnc e (Demory et al. 2016; RiddenHarper et al. 2016). We conclude that induction heating coupled with the high surface temperature can be an important driver of volcanic activity on rocky super-Earths with masses exceeding approximately four Earth masses. For very close-in planets with surface temperatures exceeding $1000 \mathrm{~K}$, a thin mineral atmosphere can also form (Ito et al. 2015). One can discriminate between the two atmosphere types by investigating the atmosphere's composition, with volcanically outgassed atmospheres dominated by $\mathrm{CO}_{2}, \mathrm{CO}, \mathrm{N}_{2}, \mathrm{H}_{2}, \mathrm{H}_{2} \mathrm{O}, \mathrm{H}_{2} \mathrm{~S}, \mathrm{~S}_{2}$ (Gaillard \& Scaillet 2014), and mineral atmospheres dominated by $\mathrm{Na}, \mathrm{Mg}$, SiO, O, among others (Miguel et al. 2011; Ito et al. 2015).

In this article we did not take into account the interaction of the volcanic atmosphere with the ambient stellar wind plasma. Such interactions perturb the magnetic field in the vicinity of a planet and can alter the induction response generated in the mantle. Important effects include plasma mass loading from newly picked-up atmospheric ions, possible Alfén wave currents that can flow through the planet's ionosphere, diamagnetism from newly picked-up plasma (e.g., Khurana et al. 1998; Saur et al. 2004). Plasma interactions produce a more complicated field structure in the planet's vicinity; for instance, the field can become stronger upstream and weaker downstream. Induction effects take place and are observable even in bodies surrounded by plasma, as is the case for the Galilean satellites embedded into the flow of Jupiter's magnetospheric plasma (Zimmer et al. 2000; Khurana et al. 2011; Roth et al. 2017) and for Mars, which interacts with the solar wind flow. Recently, it has also been shown that electromagnetic induction can power a large water current in Europa's ocean (Gissinger \& Petitdemange 2019). On Mars, the induction response to variations in the interplanetary magnetic field has been measured, and has allowed us to reconstruct the conductivity profile of the planet's mantle (Civet \& Tarits 2014). On HD 3167b, inclusion of the plasma effects would likely lead to generation of a more complicated induced magnetic field inside the planet in comparison to the dipolar field considered here. A thorough investigation of these effects using MHD simulations and a wind model similar to those existing in the literature (e.g., Johnstone et al. 2015; Cohen 2017; Boro Saikia et al. 2020) is necessary to shed light on the role played by plasma effects.

Recently, Günther \& Kislyakova (2020) have observed HD3167b in-transit and out-of-transit with UVES at the VLT in order to search for the presence of lines originating from the planet's exosphere with the aim of detecting a possible volcanic or mineral atmosphere. They have searched for lines such as the $\mathrm{NaD}_{1,2}$ and CaII H\&K lines as well as numerous [S II], [S III], and [O III] lines, which are tracers of volcanic activity. They derived upper limits of the ratios of the line flux to the stellar flux. The derived upper limits were $1.5 \times 10^{-3}$ for the CaII H\&K lines and $7.2 \times 10^{-4}$ and $3.3 \times 10^{-4}$ and for the $\mathrm{NaD}_{1,2}$ lines, respectively. The fact that these upper limits are approximately equal to previous detections in $55 \mathrm{Cnc}$ e by Ridden-Harper et al. (2016) shows that not all super-Earths show these lines continuously and that they might be variable. This indicates that further observations, at different wavelengths and with different instruments such as ARIEL and JWST, are necessary.

Acknowledgements. KK acknowledges the support by Austrian Science Fund (FWF) NFN project S116-N16 and subproject S11604-N16. The authors thank the Erwin Schrödinger Institute (ESI) of the University of Vienna for hosting the meetings of the Thematic Program "Astrophysical Origins: Pathways from Star Formation to Habitable Planets" and Europlanet for providing additional support for this program.

\section{References}

Boro Saikia, S., Lueftinger, T., Jeffers, S. V., et al. 2018, A\&A, 620, L11 Boro Saikia, S., Jin, M., Johnstone, C. P., et al. 2020, A\&A, 635, A178 Civet, F., \& Tarits, P. 2014. Earth Planets Space, 66, 85

Cohen, O. 2017, ApJ, 835, 220

Dalal, S., Hébrard, G., Lecavelier des Étangs, A., et al. 2019, A\&A, 631, A28 Demory, B.-O., Gillon, M., Madhusudhan, N., \& Queloz, D. 2016, MNRAS, 455,2018

Dorn, C., Noack, L., \& Rozel, A. B. 2018, A\&A, 614, A18 
K. Kislyakova and L. Noack: Induction heating as a driver of volcanic activity

Edwards, B., Mugnai, L., Tinetti, G., Pascale, E., \& Sarkar, S. 2019, AJ, 157, 242

Fares, R., Moutou, C., Donati, J.-F., et al. 2013, MNRAS, 435, 1451

Fares, R., Bourrier, V., Vidotto, A. A., et al. 2017, MNRAS, 471, 1246

Gaillard, F., \& Scaillet, B. 2014, Earth Planet. Sci. Lett., 403, 307

Gandolfi, D., Barragán, O., Hatzes, A. P., et al. 2017, AJ, 154, 123

Gissinger, C., \& Petitdemange, L. 2019, Nat. Astron., 3, 401

Godolt, M., Tosi, N., Stracke, B., et al. 2019, A\&A, 625, A12

Güdel, M. 2007, Liv. Rev. Sol. Phys., 4, 3

Günther, E. W., \& Kislyakova, K. G. 2020, MNRAS, 491, 3974

Ito, Y., Ikoma, M., Kawahara, H., et al. 2015, ApJ, 801, 144

Johnstone, C. P. 2012, Ph.D. Thesis, University of St Andrews, UK

Johnstone, C. P., Güdel, M., Lüftinger, T., Toth, G., \& Brott, I. 2015, A\&A, 577, A27

Khurana, K. K., Kivelson, M. G., Stevenson, D. J., et al. 1998, Nature, 395, 777 Khurana, K. K., Jia, X., Kivelson, M. G., et al. 2011, Science, 332, 1186

Kislyakova, K. G., Noack, L., Johnstone, C. P., et al. 2017, Nat. Astron., 1, 878

Kislyakova, K. G., Fossati, L., Johnstone, C. P., et al. 2018, ApJ, 858, 105

Lang, P., Jardine, M., Donati, J.-F., Morin, J., \& Vidotto, A. 2012, MNRAS, 424, 1077

Lenardic, A., Jellinek, A., \& Moresi, L.-N. 2008, Earth Planet. Sci. Lett., 271, 34

McEwen, A. S., Keszthelyi, L., Spencer, J. R., et al. 1998, Science, 281, 87
Miguel, Y., Kaltenegger, L., Fegley, B., \& Schaefer, L. 2011, ApJ, 742, L19 Morin, J., Donati, J.-F., Petit, P., et al. 2010, MNRAS, 407, 2269

Noack, L., Rivoldini, A., \& Van Hoolst, T. 2016, Int. J. Adv. Syst. Meas., 9, 66 Noack, L., Rivoldini, A., \& Van Hoolst, T. 2017, Phys. Earth Planet. Inter., 269, 40

Ortenzi, G., Noack, L., Sohl, F., et al. 2019, Nat. Sci. Rep., submitted

Parkinson, W. D. 1983, Introduction to Geomagnetism (Scottish Academic Press Ltd)

Ridden-Harper, A. R., Snellen, I. A. G., Keller, C. U., et al. 2016, A\&A, 593, A129

Roberts, J. H., \& Nimmo, F. 2008, Icarus, 194, 675

Roth, L., Saur, J., Retherford, K. D., et al. 2017, J. Geophys. Res. (Space Phys.), 122,1903

Saur, J., Neubauer, F. M., Connerney, J. E. P., Zarka, P., \& Kivelson, M. G. 2004 , in Plasma interaction of Io with its plasma torus, eds. F. Bagenal, T. E. Dowling, \& W. B. McKinnon, 1, 537

Tackley, P. J., Ammann, M., Brodholt, J. P., Dobson, D. P., \& Valencia, D. 2013, Icarus, 225, 50

Vidotto, A. A., Gregory, S. G., Jardine, M., et al. 2014, MNRAS, 441, 2361

Xu, Y., Shankland, T. J., \& Poe, B. T. 2000, J. Geophys. Res., 105, 27

Yoshino, T., \& Katsura, T. 2013, Annu. Rev. Earth Planet. Sci., 41, 605

Yoshino, T., Manthilake, G., Matsuzaki, T., \& Katsura, T. 2008, Nature, 451, 326

Zimmer, C., Khurana, K. K., \& Kivelson, M. G. 2000, Icarus, 147, 329 